\title{
Skalare Kern-Elektronen-Wechselwirkungen zwischen verschiedenen Molekülen in Lösungen
}

\author{
W. MÜlLer-W \\ Max-Planck-Institut für Chemie (Otto-Hahn-Institut), Mainz \\ (Z. Naturforschg. 21 a, 153-157 [1966] ; eingegangen am 2. September 1965)
}

Herrn Professor J. Mattauch zum 70. Geburtstag gewidmet

\begin{abstract}
The dynamic polarization of the ${ }^{19} \mathrm{~F}$ nuclei in free radical solutions of $\mathrm{C}_{6} \mathrm{~F}_{6}, \mathrm{C}_{6} \mathrm{H}_{5} \mathrm{~F}$ and $\mathrm{C}_{6} \mathrm{H}_{4} \mathrm{~F}_{2}$ is much less negative than expected from investigations of the proton polarization in similar systems. In the limits of "extreme narrowing" the enhancement factor is of the order of -100 instead of -350 ; in strong magnetic fields it is even posiitve. Data obtained in fields of $16.2,176$ and 3230 gauss are interpreted in terms of a combined dipole-dipole and scalar coupling between nuclear and electron spins. For the time dependence of the dipolar interaction the model of an independent "translational" random diffusion of spin-carrying molecules is used whilst the scalar interaction is described by a spectral function proportional to $\tau_{\mathrm{Sk}} /\left(1+\omega^{2} \tau_{\mathrm{Sk}}{ }^{2}\right)$. The results are compared with measurements of Richards in strong magnetic fields and with theoretical models of HUBBaRD for the scalar interaction. Diffusional and scalar correlation times, the relative contribution of the scalar interaction and the order of the coupling constant are calculated.
\end{abstract}

Zum Studium von Flüssigkeitsbewegungen und Lösungseigenschaften eignen sich besonders gut magnetische Resonanzmethoden. Die Kern- und Elektronenresonanzspektren hängen in charakteristischer Weise von den magnetischen Wechselwirkungen zwischen gleich- und verschiedenartigen Spins in den Flüssigkeitsmolekülen ab. Insbesondere ergeben Messungen der Relaxationszeiten und der dynamischen Polarisation von Kernspins Aussagen nicht nur über die Art der Wechselwirkungen, sondern auch über deren Zeitabhängigkeit. Als verhältnismäßig einfache Systeme bieten sich für solche Untersuchungen Flüssigkeiten oder Lösungen an, die nur zwei Spinsorten enthalten, welche $\mathrm{zu}$ verschiedenen Molekülen gehören. Die theoretische Behandlung wird in der Näherung des „Zwei-Spin-Systems“ relativ einfach, wenn es sich dabei um Elektronen- und Kernspins handelt. Als „Modellsysteme“ für Flüssigkeitsuntersuchungen haben sich Lösungen freier Radikale bewährt ${ }^{1-3}$, in denen die Kernspins zu den Protonen der Lösungsmittel-, die Elektronenspins zu den ungepaarten Elektronen der Radikalmoleküle gehören. Freie Radikale besitzen nahezu reinen Spinmagnetis-

* Gegenwärtige Adresse: CCR-Euratom, Ispra/Varese (Ital.).

1 J. Haupt, K. D. Kramer u. W. Müller-W Coll. Ampère 1962, North-Holland Publishing Co., Amsterdam 1963, S. 709.

2 K. D. Kramer u. W. Müller-Warmuth, Proc. XIth Coll. Ampère 1963, North-Holland Publishing Co., Amsterdam 1964 , S. 315.

3 K. D. Kramer u. W. Müller-W 19 a, 375 [1964]. mus, der $g$-Faktor ist ungefähr 2, und elektrische Wechselwirkungen gehen nicht in den HamiltonOperator ein, da nur Spin-1/2-Teilchen beteiligt sind.

Untersuchungen der Frequenz- und Temperaturabhängigkeit der dynamischen Protonenpolarisation in einer ganzen Reihe von organischen Lösungen freier Radikale haben gezeigt, daß die Ergebnisse durch einen "Translationsansatz" für die Molekülbewegung recht gut beschrieben werden können ${ }^{3-4}$. Das heißt: man kann eine weitgehend voneinander unabhängige Diffusion der Moleküle in den Lösungen annehmen und die charakteristischen Konstanten dieser Bewegung ableiten. Es war auch möglich, die Grenzen dieser Beschreibung anzugeben und Vergleiche mit anderen Flüssigkeitsparametern durchzuführen ${ }^{5}$. Ein unterschiedliches Verhalten, das auf bestimmte Solvatationseffekte schließen läßt, wurde bisher nur an Lösungen in Toluol beobachtet ${ }^{6}$. Trotzdem ist allen Ergebnissen - in Übereinstimmung mit früheren Untersuchungen des OverhauserEffektes in Radikallösungen ${ }^{7-9}$ - gemeinsam, daß zwischen den Protonen- und Elektronenspins nur Dipolwechselwirkungen existieren. Das äußert sich

4 K. D. Kramer, W. Müller-Warmuth u. J. Schindler, J. Chem. Phys. 43, 31 [1965].

5 K. D. Kramer, W. Müller-Warmuth u. N. Roth, Z. Naturforschg. 20 a, 1391 [1965].

6 G. J. KrüGER u. W. Müller-W $W_{\text {ARMuth, in }}$ Vorbereitung.

7 A. Abragam, J. Combrisson u. I. Solomon, C. R. Acad. Sci. Paris 245, 157 [1957].

8 W. Müller-W

9 J. Haupt u. W. Müller-W 1011 [1962]. 
im Experiment darin, daß der Vergrößerungsfaktor der dynamischen Kernpolarisation im Grenzfall tiefer Frequenzen und hoher Temperaturen - 329 beträgt, und da $\beta$ er mit wachsenden Frequenzen und sinkenden Temperaturen abfällt, aber niemals positiv wird.

Überraschenderweise führten Messungen der dynamischen ${ }^{19} \mathrm{~F}$-Kernpolarisation in ähnlich gebauten Lösungen mit fluorierten Lösungsmitteln zu einem ganz anderen Ergebnis. In verschiedenen Radikallösungen von $\mathrm{C}_{6} \mathrm{H}_{5} \mathrm{~F}, \mathrm{C}_{6} \mathrm{H}_{4} \mathrm{~F}_{2}$ und $\mathrm{C}_{6} \mathrm{~F}_{6}$ ergab sich anstatt des bei tiefen Frequenzen nach der Größe der magnetischen Momente zu erwartenden Vergrößerungsfaktors von -350 ein solcher von nur etwa - 100. Bei hohen Frequenzen wurde die Fluorpolarisation in einigen Fällen sogar positiv. Dieses Verhalten läßt sich mit gleichzeitig von Richards u. Mitarb. ${ }^{10}$ sowie von VAN Steenwinkel ${ }^{11}$ bei hohen Frequenzen erhaltenen Ergebnissen in Ubereinstimmung bringen. Unsere Meßergebnisse erwiesen sich als weitgehend unabhängig von der Art der gelösten freien Radikale. Der vorliegenden Arbeit sollen dennoch Ergebnisse im gleichen System zugrunde gelegt werden, wie es Richards benutzte, um für die Diskussion alle bekannten Meßdaten ausnutzen $\mathrm{zu}$ können ${ }^{12}$.

\section{Experimentelles und Meßergebnisse}

Die hier diskutierten Messungen wurden an Hexafluorbenzollösungen des freien Radikals Tri-t-butylphenoxyl bei der Temperatur von $+24{ }^{\circ} \mathrm{C}$ durchgeführt. Die Untersuchungen erfolgten in magnetischen Feldern von 16,2, 176 und 3230 Gauss. Die zugehörigen Resonanzfrequenzen sind für ${ }^{19} \mathrm{~F}$ : $65 \mathrm{kHz}, 706 \mathrm{kHz}$ und $13 \mathrm{MHz}$, für die Elektronenspins $45 \mathrm{MHz}, 493 \mathrm{MHz}$ und 9,1 GHz. Die benutzten Spektrometer, die Meß- und Auswertemethodiken sind die gleichen wie bei früheren Studien der Protonenpolarisation $4,8,13,14$. Die verwendeten Lösungen waren hinreichend konzentriert, damit die Elektronenresonanz aus einer austauschverschmälerten Linie bestand und damit die Streufaktoren $f$ zwischen 0,94 und $0,98 \mathrm{zu}$ liegen kamen.

10 R. A. Dwek, J. G. Kenworthy, D. F. S. Natusch, R. E. Richards u. D. J. Shields, Proc. Roy. Soc. London, im Druck.

11 R. Van Steenwinkel, Ispra, persönliche Mitteilung.

12 Herrn Prof. Richands, Oxford, bin ich zu besonderem Dank für die freundliche Uberlassung einer Probe $\mathrm{C}_{6} \mathrm{H}_{6} /$ Tri-tbutylphenoxyl verpflichtet.
Abb. 1 zeigt das Ergebnis. Aufgetragen als Funktion der Elektronenresonanzfrequenz ist der KernElektronen-Kopplungsparameter $\varrho$, der sich aus den gemessenen Vergrößerungsfaktoren $P / P_{0}$ nach

$$
\frac{P}{P_{0}}=1-\varrho f s \cdot \gamma_{S} / \gamma_{I}
$$

ergibt. $f$ und $s$ wurden auf die übliche Weise ${ }^{3,9}$ aus Messungen der Kernrelaxationszeiten der Lösung und des reinen Lösungsmittels, bzw. aus Messungen bei verschiedenen Elektronenresonanz-Sättigungsleistungen ermittelt. $\gamma_{S} / \gamma_{I}$ beträgt hier 700 , wenn man die gyromagnetischen Verhältnisse des freien Elektrons und des ${ }^{19} \mathrm{~F}$-Kerns einsetzt. In die gleiche Darstellung, Abb. 1, wurden die Meßergebnisse von Richards eingetragen, die Magnetfeldern von 3300 bzw. 12500 Gauss entsprechen ${ }^{10}$.

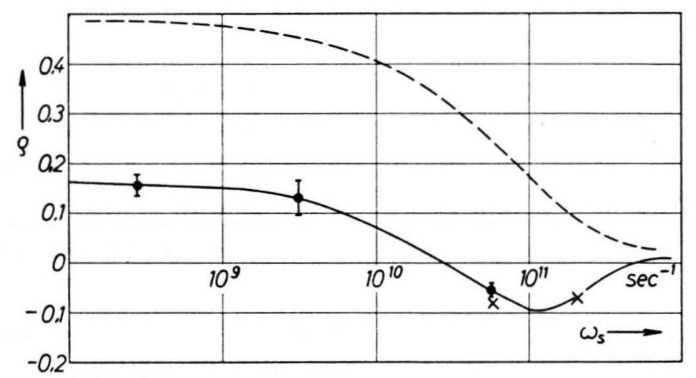

Abb. 1. Meßergebnisse der dynamischen ${ }^{19} \mathrm{~F}$-Kernpolarisation in Hexafluorbenzol-Lösungen bei $24^{\circ} \mathrm{C}$. Der gezeichnete Kurvenverlauf ist das Ergebnis der im Text gegebenen Interpretation. Zum Vergleich zeigt die gestrichelte Kurve die in Lösungen freier Radikale in Benzol beobachtete Frequenzabhängig. keit der Protonenpolarisation. $x$ Meßpunkte von Richards et al. ${ }^{10}$.

Der gestrichelte Kurvenverlauf in Abb. 1 ist das Ergebnis von Messungen der dynamischen Protonenpolarisation an gewöhnlichen Benzollösungen bei der gleichen Temperatur. Ebenso wie in $\mathrm{C}_{6} \mathrm{~F}_{6}$-Lösungen hängt in $\mathrm{C}_{6} \mathrm{H}_{6}$-Lösungen der Verlauf nur wenig von der Art des freien Radikals ab. Wenn in Benzol und Hexafluorbenzol die gleichen Wechselwirkungsmechanismen zwischen Kern- und Elektronenspins wirksam wären, hätte man für den Overhauser-Effekt der ${ }^{19} \mathrm{~F}$-Kerne die gleiche Frequenzabhängigkeit erwartet (gestrichelte Kurve), oder eine solche, die infolge einer etwas geänderten Korrelationszeit der Molekül-

13 H. Grützediek, K. D. Kramer u. W. Müller-W Sci. Instr. 36, 1418 [1965].

14 Meinen Mitarbeitern H. Grützediek und J. Schindler danke ich für die Durchführung von Messungen bei 176 und 3230 Gauss. 
bewegung in der Frequenzachse leicht parallel verschoben ist. Die durch die Meßpunkte gelegte Kurve ist das Ergebnis der nachfolgenden Diskussion.

\section{Diskussion}

Die kleinen Vergrößerungsfaktoren bei tiefen Frequenzen und die negativen $\varrho$-Werte bei hohen Frequenzen können nur durch eine zeitabhängige skalare Kopplung erklärt werden, die neben den Dipolwechselwirkungen zwischen den Kern- und Elektronenspins wirksam ist. Das bedeutet, daß sich im Verlaufe der Flüssigkeitsbewegung Wellenfunktionen von zu verschiedenen Molekülen gehörenden Elektronen und ${ }^{19} \mathrm{~F}$-Kernen zeitweise überlappen. Das ist insofern überraschend, als ein solcher Effekt für Protonen in ähnlich gebauten Systemen bisher nicht beobachtet wurde, und man als Folge einer Fluorierung zunächst keinen so grundlegenden Wandel in der Lösungsstruktur erwartet hätte.

Die Voraussetzungen für die Berechnung von $\varrho$ sind an anderer Stelle ${ }^{3,5}$ angegeben. In den Wechselwirkungsoperator war bereits eine skalare Wechselwirkung einbezogen ${ }^{3}$, so $\mathrm{da} ß$ sich schließlich unter den in diesen Arbeiten präzisierten Gültigkeitsbedingungen und Bezeichnungen ergab - vgl. ${ }^{5}$, Gl. (5) -

$$
\varrho=\frac{5 J_{\mathrm{D}}\left(\omega_{S}\right)-5 J_{A}\left(\omega_{S}\right) /\left(4 \pi \gamma_{S^{2}} \gamma_{I}{ }^{2} \hbar^{2}\right)}{7 J_{\mathrm{D}}\left(\omega_{S}\right)+3 J_{\mathrm{D}}\left(\omega_{I}\right)+5 J_{A}\left(\omega_{S}\right) /\left(4 \pi \gamma_{S^{2}} \gamma^{2} \hbar^{2}\right)} .
$$

Alle bisher erhaltenen Ergebnisse der dynamischen Protonenpolarisation konnten mittels (2) und $J_{A}=0$ beschrieben werden (gestrichelter Verlauf in Abb. 1). Für die Spektraldichtefunktionen der durch die Molekularbewegung statistisch schwankenden DipolDipol-Wechselwirkungen, $J_{\mathrm{D}}$, wurde zur Erklärung der experimentellen Ergebnisse

$$
J_{\mathrm{D}}(\omega)=\frac{6 N_{S} \tau_{\mathrm{c}}}{d^{3}} f\left(\omega \tau_{\mathrm{c}}\right)
$$

gesetzt $\left[N_{S}=\right.$ Elektronenspinkonzentration, $d=$ minimal möglicher Abstand der Spins]. $f\left(\omega \tau_{c}\right)$ ist eine maschinell ausgewertete Funktion der Frequenz mit einer Korrelationszeit $\tau_{\mathrm{c}}$, die von Pfeifer ${ }^{15}$ explizit beechnet wurde, und die in ${ }^{3}$ dargestellt ist. (3) ergibt sich bei einer im wesentlichen translatorischen

15 H. Pfeifer, Ann. Phys. Leipzig 8, 1 [1961].

16 A. Abragam, Principles of Nuclear Magnetism, Clarendon Press, Oxford 1961, Chapt. VIII.
Diffusionsbewegung der die Spins tragenden Moleküle. In Ausnahmefällen ist dem „Translationsanteil“ (3) ein schwacher "Rotationsanteil“ beigemischt ${ }^{5,6}$. Wir wollen hier aber diesen möglichen schwachen Anteil vernachlässigen und den Dipolanteil an der dynamischen Polarisation allein durch (3) beschreiben.

Die dynamische Kernpolarisation reagiert nach (2) außerordentlich empfindlich auf die Art der Wechselwirkungen, weil Dipolkopplungen zu negativen Polarisationen $(\varrho>0)$, Skalarkopplungen aber zu positiven $(\varrho<0)$ führen. Als einfachstes Modell für die Skalarkopplung $A \mathbf{S}$ I bietet sich ein solches an, bei dem $A$ eine statistische Funktion der Zeit ist, die nur zwei Werte hat: nämlich $A$, wenn die Moleküle, zu denen $S$ und $I$ gehören, zusammenkleben (so daß die Wellenfunktionen sich überlappen), und 0, wenn nicht. Es ergibt sich dann für die Spektraldichte die früher ${ }^{3,5}$ angesetzte explizite Form,

$$
J_{A}(\omega)=W_{A}(A / \hbar)^{2} \frac{2 \tau_{\mathrm{Sk}}}{1+\omega^{2} \tau_{\mathrm{Sk}}{ }^{2}} .
$$

Der Ansatz (4) wurde wiederholt zur Beschreibung von skalaren Kopplungsmechanismen benutzt, die durch chemischen Austausch oder durch Elektronenspinrelaxation zeitmoduliert sind $\mathbf{1 6}^{\mathbf{1 7}}$. Die Korrelationszeit $\tau_{\mathrm{Sk}}$ bedeutet hier einen zeitlichen Mittelwert für die statistische Zeitvariable, die die Dauer des "Zusammenklebens" der Moleküle mißt. Zur Überlagerung der Relaxationsmechanismen (3) und (4) $m u ß$ angenommen werden, daß für das Gesamtsystem der Fluorkerne durch einen genügend schnellen Austausch der gekoppelten und ungekoppelten Kerne eine einheitliche Relaxationszeit existiert. $\tau_{\mathrm{Sk}}$ wird kurz gegenüber dem „skalaren Anteil“ dieser Relaxationszeit angesetzt und die zu $\tau_{\mathrm{Sk}}$ gehörende mittlere Aufenthaltswahrscheinlichkeit $W_{A} \ll 1$. In jedem Augenblick soll sich im Mittel nur ein Bruchteil der Fluorkerne in Kontaktwechselwirkung befinden, so daß die Diffusionsbewegung der Mehrzahl der Moleküle dadurch nur unwesentlich gestört wird. Nur unter diesen Voraussetzungen läßt sich ein übersichtliches Ergebnis ableiten. Mit (3) und (4) ergibt dann (2)

$$
\varrho=\frac{5-5 K \frac{\tau_{\mathrm{Sk}}}{\tau_{\mathrm{c}}} \frac{1 /\left(1+\omega_{S}{ }^{2} \tau_{\mathrm{Sk}}{ }^{2}\right)}{15 / 2 f\left(\omega_{\mathrm{S}} \tau_{\mathrm{c}}\right)}}{7+3 \frac{f\left(\omega_{I} \tau_{\mathrm{c}}\right)}{f\left(\omega_{\mathrm{S}} \tau_{\mathrm{c}}\right)}+5 K \frac{\tau_{\mathrm{Sk}}}{\tau_{\mathrm{c}}} \frac{1 /\left(1+\omega_{\mathrm{S}}{ }^{2} \tau_{\mathrm{Sk}}{ }^{2}\right)}{15 / 2 f\left(\omega_{S} \tau_{\mathrm{c}}\right)}} .
$$

17 N. Bloembergen, J. Chem. Phys. 27, 572 [1957]. - H. PfeiFER, Z. Naturforschg. 17 a, 279 [1962]. - R. H HUSSER u. F. Noack, Z. Phys. 182, 93 [1964]. - R. Sperling u. H. P Feifer, Z. Naturforschg. 19 a, 1342 [1964] u. a. 
Die dimensionslose Größe $K$ mißt den Anteil der Skalarkopplung

$$
K=\frac{5 W_{A} d^{3}(A / \hbar)^{2}}{8 \pi \gamma_{S}^{2} \gamma I^{2} \hbar^{2} N_{S}} .
$$

Die bestmögliche Anpassung der Frequenzabhängigkeit von (5) an die Meßpunkte von Abb. 1 führt zu folgenden Zahlenwerten (in Abb. 1 eingezeichneter Kurvenzug)

$$
\begin{aligned}
K & =0,8( \pm 0,05), \\
\tau_{\mathrm{c}} & =(0,9 \pm 0,1) \cdot 10^{-11} \mathrm{sec}, \\
\tau_{\mathrm{Sk}} & =(0,7 \pm 0,1) \tau_{\mathrm{c}} .
\end{aligned}
$$

Die angegebenen Grenzen ergeben sich unter der Voraussetzung der Gültigkeit von (5) und aus der Berücksichtigung der Fehlergrenzen der Meßpunkte bei der Anpassung an (5). Die Größenordnungen dieser Werte erscheinen vernünftig. Man hätte allerdings eine Korrelationszeit $\tau_{\mathrm{c}}$ der Translationsdiffusion erwartet, die eher ein klein wenig länger als die von Lösungen des gleichen Radikals in Benzol ist. Letztere wurde zu 2,5 $10^{-11} \mathrm{sec}$ bestimmt. Doch kann dieser - bei der Grobheit des Modells geringe - Unterschied damit zusammenhängen, daß die translatorische Diffusionsbewegung durch die Austauschwechselwirkungen gestört wird. Komplizierend wirkt sich natürlich aus, daß $\tau_{\mathrm{c}}$ und $\tau_{\mathrm{Sk}}$ die gleiche Größenordnung besitzen.

Aus $K$ läßt sich die Größenordnung der Skalarkopplungskonstanten $A$ abschätzen. Aus Messungen der Relaxationszeit ${ }^{18}$ ergab sich für den kleinstmöglichen Abstand der Spins bei der Dipolwechselwirkung, $d=3,9 \AA$. Wenn man aus Mangel an besseren Argumenten $W_{A}=N_{S} / N_{I}$ setzt, d. h. annimmt, $\mathrm{da} ß \mathrm{im}$ Mittel in jedem Augenblick ein Fluorkern mit einem Radikal in Kontakt steht $\left(N_{I}=3,1 \cdot 10^{22} \mathrm{~cm}^{-3}\right.$, Konzentration der Fluorkerne), dann folgt mit $K=0,8$ aus (6)

$$
A / h \approx 3,4 \mathrm{MHz} .
$$

Die hier durchgeführten Überlegungen erklären übrigens auch auf einfache Weise, weshalb sich der Skalarkopplungsanteil, der die dynamische FluorKernpolarisation so stark beeinflußt, bei Untersuchungen der Frequenzabhängigkeit der SpinGitter-Relaxationszeit kaum bemerkbar macht ${ }^{18}$. Die durch Wechselwirkungen mit den Elektronen bedingte Relaxationsrate der ${ }^{19} \mathrm{~F}$ läßt sich unter den

18 V. Printz, Dissertation, Universität Mainz, Veröffentlichung in Vorbereitung. gleichen Voraussetzungen, die zu (5) führten, als Summe zweier Relaxationsraten darstellen:

$$
\begin{aligned}
& \frac{1}{T_{1}^{\prime}}=\frac{1}{T_{1} \mathrm{D}}+\frac{1}{T_{1} \mathrm{Sk}} \\
& =K_{1}\left[7 \tau_{\mathrm{c}} f\left(\omega_{S} \tau_{\mathrm{c}}\right)+3 f\left(\omega_{I} \tau_{\mathrm{c}}\right)+\frac{2}{3} K \frac{\tau_{\mathrm{Sk}}}{1+\omega^{2} \tau_{\mathrm{Sk}}{ }^{2}}\right] \\
& \text { mit } \quad K_{1}=\frac{6 \pi}{5} \gamma_{S^{2}} \gamma_{I}^{2} \hbar^{2} N_{\mathrm{S}} / d^{3} .
\end{aligned}
$$

Mit den oben angegebenen Werten für $K, \tau_{\mathrm{c}}$ und $\tau_{\mathrm{Sk}}$ ergibt sich, daß der skalare Term bei der Frequenz Null etwa 28\% ausmacht, dann bis auf etwa $45 \%$ wächst und bei $\omega_{S}=2,2 \cdot 10^{11} \mathrm{~s}^{-1}$ noch $26 \%$ beträgt. Das heißt, wegen $\tau_{\mathrm{c}} \approx \tau_{\mathrm{Sk}}$ ändert sich der Verlauf von $1 / T_{1}{ }^{\prime}(\omega)$ kaum, der Skalaranteil entgeht der Beobachtung, und man bestimmt ein falsches $K_{1}$.

\section{Theorie von Hubbard}

Nach der Diskussion des letzten Abschnitts bleibt die Frage offen, inwieweit die Beschreibung der skalaren Wechselwirkungen modellabhängig ist. Da Meßdaten bei Frequenzen vorliegen, die sich um drei Größenordnungen unterscheiden, erwartet man eine gewisse Ansprechempfindlichkeit auf Feinheiten des Modells. Insbesondere bietet sich die Möglichkeit, die Ergebnisse einer kürzlich von HubBard durchgeführten theoretischen Untersuchung ${ }^{19}$ über skalare Kern-Elektronen-Wechselwirkungen zu prüfen.

HubBard geht von zwei Hypothesen aus, die er „sticking model“ und „diffusion model“ nennt. Die erste führt zu dem üblicherweise benutzten Ansatz (4), allerdings mit $\tau_{\mathrm{Sk}} \ll \tau_{\mathrm{c}}$. Mit dieser Einschränkung vermeidet man die in Abschn. 2 erwähnten Komplikationen, die durch Störungen der Moleküldiffusion infolge des gelegentlichen „Zusammenklebens" von Radikal- und Lösungsmittelmolekülen entstehen. Im Diffusionsmodell wird die skalare Wechselwirkungsenergie zwischen zwei Molekülen $i$ und $j$ proportional $A\left(d / r_{i j}\right) e^{-\lambda\left(r_{i j}-d\right)}$ angesetzt (Konstante $\lambda$ mit $\lambda d \gg 1)$. Die Zeitabhängigkeit ergibt sich hier aus den Änderungen des Abstandes $r_{i j}$ zwischen Kern und Elektron, wenn die Moleküle in der Flüssigkeit diffundieren. Hubbard zeigt im einzelnen, daß eine solche Wechselwirkung von sehr kurzer Reichweite durchaus die Kernrelaxation beeinflussen kann.

19 P. S. Hubbard, Proc. Roy. Soc. London, im Druck. 
Zum Vergleich der einzelnen Hypothesen wurden in Abb. 2 die Meßergebnisse - einschließlich derer von RichaRds - noch einmal in einem vergrößerten Maßstab dargestellt. Die kräftig durchgezeichnete Kurve (I) ist das Ergebnis der Diskussion von Abschn. 2. Der strichpunktierte Verlauf (II) entspricht der bestmöglichen Anpassung, wenn man $\tau_{\mathrm{Sk}} \ll \tau_{\text {c }}$ annimmt; $K$ hat dabei den gleichen Wert

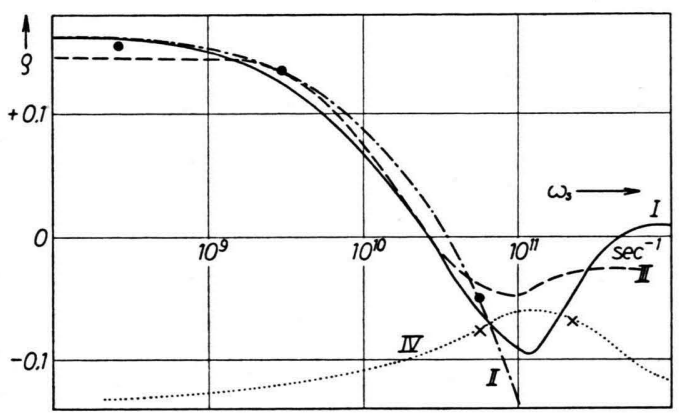

Abb. 2. Anpassung der Parameter verschiedener Modelle zur Beschreibung der skalaren Wechselwirkungen an die Meßergebnisse: (I) vorliegende Arbeit, (II) Hubbards „sticking model“, (III) Hubbards „diffusion model“, (IV) Interpretation von Richards nach dem Diffusionsmodell vor Kenntnis dieser Arbeit.

(bei tiefen Frequenzen ist die Spektralfunktion sowieso kaum frequenzabhängig), $\tau_{\mathrm{c}}$ ist $6 \cdot 10^{-12} \mathrm{sec}$. Eine möglichst gute Anpassung des Diffusionsmodells an alle Meßpunkte führt zu der gestrichelten Kurve (II) mit $\tau_{\mathrm{c}}=1,5 \cdot 10^{-10} \mathrm{sec}$ und $\xi=8,5$. Der von Hubbard eingeführte Parameter $\xi$ hat eine ähnliche Bedeutung wie $K$ in (6),

$$
\xi=\frac{100(A / \hbar)^{2} \cdot d^{4}}{\gamma S^{2} \gamma I^{2} \hbar^{2} \lambda^{2}} .
$$

Die einzelnen Kurven in Abb. 2 zeigen, daß leicht Fehlinterpretationen möglich sind, wenn nur Meßdaten in einem begrenzten Frequenzbereich zur Verfügung stehen. Ohne Berücksichtigung der Messung von RichaRds bei $\omega_{S}=2,2 \cdot 10^{11} \mathrm{sec}^{-1}$ könnte man kaum eine Entscheidung zwischen (I), (II) und (III) treffen. Noch deutlicher wird dies, wenn man nur die Ergebnisse bei hohen Frequenzen berücksichtigt. Richards und Mitarb. interpretieren ihre Messungen durch den punktierten Kurvenverlauf (IV) $(\xi=20$, $\left.\tau_{\mathrm{c}}=6,5 \cdot 10^{-12} \mathrm{sec}\right)$ und treffen eine Entscheidung zugunsten des HubBardschen Diffusionsmodells.

Bei Berücksichtigung der bis jetzt bekannten experimentellen Ergebnisse fällt nach Abb. 2 eine Entscheidung zwischen den hier diskutierten Modellen nicht schwer. Hinzu kommt, daß nur Kurve I zu einer Korrelationszeit $\tau_{\mathrm{c}}$ führt, die halbwegs vernünftig erscheint. Für eine weitere Verfeinerung des Modells fehlen aber dennoch mehr experimentelle Ergebnisse, und vor allem sollte die Temperaturabhängigkeit der dynamischen Fluorpolarisation bei den einzelnen Frequenzen untersucht werden.

Die Interpretation hat keine Antwort auf die Frage gegeben, warum zwischen Fluorkernen und Elektronenspins, nicht aber zwischen Protonen und Elektronen, skalare Wechselwirkungen bestehen, wenn beide Spinsorten zu verschiedenen Molekülen gehören. Es ist sicher interessant, diesen Effekt bei anderen Kernen zu studieren.

Den Herren Prof. Dr. R. E. Richards, F. R. S., Oxford, und Prof. Dr. Paul S. Hubbard, Chapel Hill (North Carolina), z. Zt. Oxford, bin ich zu besonderem Dank für die Zusendung einer Kopie ihrer Manuskripte vor Erscheinen der Veröffentlichung verpflichtet. 